\title{
Stochastic Motion of Heavy Quarks in Holography: A Theory-Independent Treatment
}

\section{Dimitrios Giataganas*}

Physics Division, National Center for Theoretical Sciences, National Tsing-Hua University, Hsinchu, 30013, Taiwan

E-mail: dimitrios.giataganasects.nthu.edu.tw

\begin{abstract}
Stochastic dynamics play a central role in strongly coupled phenomena. We present and review a theory independent approach in holography to study such phenomena. We firstly argue that the heavy quark diffusion occurs in realistic strongly coupled systems. Then we analyze the quantum and thermal fluctuation, dissipation and the corresponding Brownian motion of a heavy particle in such environments for a wide class of theories. The holographic study is based on the properties of the straight string fluctuations. The observables and coefficients associated with the stochastic motion depend on a single parameter which encodes the properties of the different theories. Moreover, certain Dp-brane fluctuations can be mapped one-to-one to the string fluctuations and therefore the stochastic brane observables can be read from the string ones.

Then we review the Langevin diffusion of a moving heavy quark in generic thermal holographic theories. The analysis is based on the properties of the trailing string and its fluctuations. The string world-sheet has a black hole horizon and the quarks feels an effective temperature different than the environmental one. The formulas of the effective temperature, the drag force on the particle and the Langevin coefficients are given in terms of the background metric elements readily applicable to any theory. At the end we comment on the backreaction effects on the medium and present results of the Monte Carlo simulations.
\end{abstract}

Corfu Summer Institute 2017 'School and Workshops on Elementary Particle Physics and Gravity' 2-28 September 2017

Corfu, Greece

\footnotetext{
* Speaker.
} 


\section{Brief introduction to Langevin Dynamics}

Non-equilibrium systems evolving towards equilibrium exist everywhere in nature and have a wide range of applications, from soft matter and biophysics to strongly coupled phenomena in the most extreme phases of matter. The point of converging of such phenomena is the Brownian motion [1], the irregular motion of mesoscopic particles that are large enough to admit a hydrodynamic type of coarse-graining, but small enough to exhibit thermal fluctuations in a liquid environment caused by random microscopic interactions with the particles of the medium. The implications of Brownian motion were very important even from the initial stages of their theoretical study, verifying at the time the existence of atomic nature of matter and providing a good estimation of the Avogadro's constant $[2,3,4,5]$. Shortly after these developments, the probabilistic description was introduced to lay down the foundation of non-equilibrium statistical mechanics with the Langevin and Fokker-Planck equations. Since then there is a continuous interest on theoretical developments and new applications in all quantitative sciences, making the topic, a slow evolving revolution $[6,7,8,9]$.

The fluctuation and dissipation effects can be realized in the classical Langevin equation

$$
\dot{p}_{i}(t)=-\eta_{D} p_{i}(t)+\xi_{i}(t),
$$

where $p$ is the momentum of the particle, $\eta_{D}$ is the so called friction coefficient or the momentum drag coefficient and $\xi$ is the random force. These two competing phenomena are realized in nature by the friction force corresponding to dissipation, representing the energy transfer to the environment, and the noise of the environment which contributes the right amount of energy to the system through the fluctuations so that it evolves towards equilibrium. The physical picture described can be formulated as a theorem: the fluctuation-dissipation theorem relating the magnitudes of these phenomena while at equilibrium.

The theoretical developments are based on natural assumptions, mainly on separation of the timescales of the phenomena involved. These are the larger relaxation time needed for the particle to thermalize compared to the smaller collision timescale. The random force then has the following properties: i) for timescales larger the collision time the force is stochastic with zero mean value, ii) the stochastic force is uncorrelated to itself and is translationally invariant, property triggered by the equilibrated homogeneous environment, iii) the statistical properties of the random force obey the time-translational invariance. These are summarized to the following relations

$$
\langle\xi(t)\rangle=0, \quad\left\langle\xi(t) \xi\left(t^{\prime}\right)\right\rangle=\kappa \delta\left(t-t^{\prime}\right)
$$

where $\kappa$ is a constant measuring the degree of correlation and represents the mean squared momentum per unit of time. A particle experienced such a random force undergoes a random independent displacement generated by the integral

$$
\int_{0}^{t} \xi\left(t^{\prime}\right) d t^{\prime}=\int_{0}^{t_{1}} \xi\left(t^{\prime}\right) d t^{\prime}+\int_{t_{1}}^{t_{2}} \xi\left(t^{\prime}\right) d t^{\prime}+\ldots
$$

which can be thought as a summation of independent terms, each one drawn from the same distribution, resulting to a total integral obeying a normal distribution with zero mean. By applying the 
central limit theorem of statistics one may obtain directly $\left\langle\dot{x}^{2}\right\rangle \sim t$. Alternatively, the solution of the stochastic equation for $\tau \gg \eta_{D}^{-1}$ is

$$
p_{i}(t)=\int_{-\infty}^{t} d t^{\prime} e^{\eta_{D}\left(t^{\prime}-t\right)} \xi_{i}\left(t^{\prime}\right), \quad\left\langle p^{2}\right\rangle=\int^{t} d t_{1} d t_{2} e^{\eta_{D}\left(t_{1}+t_{2}\right)}\left\langle\xi_{i}\left(t_{1}\right) \xi_{i}\left(t_{2}\right)\right\rangle=\frac{3 \kappa}{2 \eta_{D}} .
$$

From the equipartition theorem the typical thermal momentum reads $p \sim \sqrt{M T}$ and therefore the drag coefficient is related to temperature (1.4) as

$$
\eta_{D}=\frac{\kappa}{2 M T} .
$$

To introduce the diffusion coefficient we compute the mean squared position of the particle from (1.1) at later time and by using again the equipartition theorem we get

$$
\left\langle x_{i}(t) x_{j}(t)\right\rangle=2 D t \delta_{i j}, \quad\left\langle x^{2}(t)\right\rangle=\frac{1}{M^{2}} \int^{t} d t_{1} d t_{2}\left\langle p\left(t_{1}\right) p\left(t_{2}\right)\right\rangle=\frac{6 T t}{M \eta_{D}},
$$

relating the diffusion constant with respect to the drag coefficient (1.5) and to the mean squared momentum transfer as

$$
D=\frac{T}{M \eta_{D}}=\frac{2 T^{2}}{\kappa} .
$$

\subsection{Justification of the Heavy Quark Diffusion in the Quark-Gluon Plasma}

The task of modeling the heavy quark interaction in thermal environment is amenable to similar diffusion treatment we have just described $[10,11,12,13]$. The role of the heavy test particle of the previous paragraph undergoing a Brownian motion, is played by a heavy quark in an environment of a light-particle fluid. The charm and bottom quark masses are much larger than the temperature and the constituent masses of the equilibrated Quark-Gluon-Plasma (QGP) environment, providing a good separation between the relaxation and the collision times. Let us justify this by the following estimations.

The typical non-relativistic thermal momentum of a heavy quark with mass $\mathrm{M}$ with $M \gg T$, is $p^{2} \sim M T \gg T^{2}$ resulting to the low velocity $v^{2} \sim T / M \ll 1$. The typical square momentum transfer from the medium for hard collisions is of order $Q^{2} \sim T^{2}$ from the equipartition theorem, therefore we eventually have $p \gg Q$. As a result a large number of collisions of order $M / T$ is required to change the momentum by a factor of order one. Therefore, the interaction of the heavy quark with the medium can be formulated with uncorrelated momentum kicks, and the Boltzmann equation in momentum transfer can be expanded to the Fokker-Planck equation of heavy quark diffusion, in the medium of the QGP. We can further estimate $\alpha_{S} \simeq 0.5$ and $M / T=7$ to obtain the drag coefficient with respect to the diffusion constant $\eta_{D}^{-1} \simeq 7 D$.

Therefore, the Brownian motion and the dissipation phenomena provide direct observables for the heavy quarks in strongly coupled theories, capable to reveal potential interesting properties of the fundamental interactions. In a sense the heavy quark interactions can be thought as encoded in the transport coefficients, which in principle are related to the scattering matrix elements on light partons in the QGP.

One way to study the heavy quark diffusion is by perturbation theory [10], where the medium is approximated as a weakly interacting system of quark and gluons, treatment that is not reliable 
in conditions realized at heavy ion colliders. Non-perturbative interactions can be captured by effective resonance models [11] allowing to compute certain resonances related to the diffusion dynamics, or by methods of holography which we discuss in this review.

\section{Fluctuation and Dissipation in Holography: A Unified Approach}

In this section we focus mostly on the description of heavy quark diffusion in the context of the gauge/gravity duality reviewing mainly the unified study scheme developed in [14]. Previous works initializing the ideas on AdS and Lifshitz spacetime include [15, 16, 17, 18, 19]. While other relevant works include $[20,21,22,23,24,25,26,27,28,29,30]$. In the next section we will study the fluctuations and the energy loss of a moving heavy quark.

To present the general picture, let us introduce the gravity dual theory in string frame

$$
d s^{2}=-g_{00}(r) d x_{0}^{2}+g_{r r}(r) d r^{2}+\sum_{i=1}^{d} g_{i i}(r) d x_{i}^{2},
$$

with $\lim _{r \rightarrow \infty} g_{i i}(r)=\infty$, such that the boundary is at $r=\infty$. $d$ are the space dimensions and the metric is diagonal. The dual field theory lives on the spacetime spanned by $\left(x_{0}, x_{i}\right)$ and $r$ is the holographic direction.

The massive heavy particle is represented by a string initiating from to the boundary spacetime, introducing therefore to the field theory extra degrees of freedom, and extending to the bulk of the space in the IR until the point $r=r_{h}$. For a thermal quantum field theory the $r_{h} \neq 0$ corresponding to the horizon of the black hole, while for a field theory at zero temperature $r_{h}=0$ and the string terminates at the deep IR. The dynamics of such strings are described by solutions of the Nambu-Goto (NG) action. Let us consider a worldsheet which extends along the $x_{1}$ direction being parametrized by $x_{1}=x_{1}(\tau, \sigma), u=\sigma, x_{0}=\tau$, where $(\tau, \sigma)$ are the worldsheet coordinates. The action is equal to

$$
S=-\frac{1}{2 \pi \alpha^{\prime}} \int d \sigma d \tau \sqrt{-\left(g_{00}+g_{11} \dot{x}_{1}^{2}\right)\left(g_{r r}+g_{11} x_{1}^{\prime 2}\right)}
$$

For a static particle one expects by symmetry arguments a straight string solution. Indeed it can be easily found that the solution to the equations of motion of the above action is $x_{1}=0$, where a space coordinate transformation is implemented to bring the origin at the position of the string.

The fluctuations of the heavy quark are realized by the dynamics of string fluctuations. For the static quark we need to consider the fluctuations around $x_{1}=0$. However, the length of the string-worldsheet in the bulk is infinite due to the infinite distance of the boundary of the space to its interior. Since the length is proportional to the particle's mass, we need to introduce a Neumann boundary condition on the location of a flavor brane $r_{b}$ close to the boundary. The boundary condition then reads $x_{1}^{\prime}\left(r_{b}\right)=0$. The fluctuations $\delta x_{1}(t, r)$ give the Nambu-Goto action in terms of the metric elements [14]

$$
S=c-\frac{1}{4 \pi \alpha^{\prime}} \int d \sigma d \tau\left(-\frac{g_{11} \sqrt{-g_{00}}}{\sqrt{g_{r r}}} \delta x_{1}^{\prime 2}+\frac{g_{11} \sqrt{g_{r r}}}{\sqrt{-g_{00}}} \delta \dot{x}_{1}^{2}\right) .
$$

The Fourier decomposed fluctuations then take the form

$$
\delta x_{1}(t, r)=\int_{0}^{\infty} h_{\omega}(r)\left(\alpha(\omega) e^{-i \omega \tau}+\alpha(\omega)^{\dagger} e^{i \omega \tau}\right),
$$


with $\alpha(\omega)^{\dagger}$ and $\alpha(\omega)$ being the creation and annihilation operators, and the mode equation reads

$$
\frac{\partial}{\partial r}\left(\frac{g_{11} \sqrt{-g_{00}}}{g_{r r}} h_{\omega}(r)^{\prime}\right)+\omega^{2} \frac{g_{11} \sqrt{g_{r r}}}{\sqrt{g_{00}}} h_{\omega}(r)=0 .
$$

\subsection{Heavy Quark Fluctuation at Zero Temperature}

It is known that in quantum physics the fluctuation and dissipation phenomena are present even at zero temperature due to vacuum fluctuations of the environment fields and the uncertainty principle. Even in the simplest case of the zero-point energy of the electromagnetic field it has been shown that the fluctuation and dissipation phenomena occur and the relevant theorems hold [31]. Perturbative methods analyzing these phenomena have also been developed to integrate out the environmental degrees of freedom by modeling them as an infinite number of simple harmonic oscillators [32, 33, 34, 35, 36, 37]. Ohmic, sub-subohmic, supra-ohmic environmental effects have been analyzed in such approaches, for example works on the latter ones include [38, 39].

The quantum fluctuations on the heavy quark in a strongly coupled environment follows the same logic. The test particle fluctuations are induced by its coupling to the gluonic fields, resulting to a non-uniform motion. The dissipation is realized by a gluonic radiation back to the medium induced by the non-canonical motion of the quark. Here we analyze the resummed physical effects of such theories using techniques of gauge/gravity duality, reviewing the generical methodology developed in [14].

To proceed to the solution of the fluctuation equations, it is necessary to consider a certain general class of theories with dual backgrounds that belong to (2.1), which we choose as

$$
g_{00}=r^{a_{0}} f(r), \quad g_{r r}=\frac{1}{r^{a_{u}} f(r)}, \quad g_{i i}=r^{a_{i}}, \quad f(r)=1, \quad r_{h}=0,
$$

where the $i$ indices count the spatial directions and $a_{i}$ are constant powers. The class of the dual field theories accommodated by the above metric includes the hyperscaling Lifshitz violating ones [40, 41, 42], the anisotropic theories [43, 44, 45, 46, 47, 48] and several other. Features of the current analysis are applicable for backgrounds with asymptotics of (2.6) like certain RG flow gravity dual solutions.

There are several reasons for choosing the form of the metric as in (2.6). Certain rescaling can bring it to a form with less number of constants $a_{i}$, however this would not make our presentation simpler, since our results are formulated in terms of a constant $v$ incorporating all the scalings of the background. Moreover, formulas derived below with the form of the chosen metric are directly applicable to any gravity background accommodated, without the need of any coordinate transformation. Finally, such choice is convenient to build on the finite temperature string fluctuation analysis and to make the mapping of the string-brane fluctuations we study in later sections.

Notice that the string fluctuations in hyperscaling violation theories at zero temperature [49] guarantees that our methods at zero temperature go through all the way for particular metrics although the theories considered here are more generic than the hyperscaling ones. These include for example the anisotropic theories with different stability and physical ranges of the parameters, compared to hyperscaling theories, allowing new features on string fluctuations. Our notation offers also a powerful advantage, since each scaling is unique, we track accurately how the different metric elements affect the observables. This is the crucial point that will allows us to identify the 
order of the Bessel function of the fluctuations as the central quantity that the stochastic observables depend exclusively on. This observation holds even in finite temperature as we will show in later sections.

For this class of the background, the mode equations (2.5) becomes

$$
\frac{\partial}{\partial r}\left(r^{a_{1}+\frac{a_{0}+a_{u}}{2}} h_{\omega}(r)^{\prime}\right)+\omega^{2} r^{a_{1}-\frac{a_{0}+a_{u}}{2}} h_{\omega}(r)=0,
$$

with a solution of type [14]

$$
h_{\omega}(r)=r^{-v \kappa} A_{\omega}\left[J_{v}(\omega \tilde{r})+B_{\omega} Y_{v}(\omega \tilde{r})\right],
$$

where $A_{\omega}$ and $B_{\omega}$ are constants and

$$
\tilde{r}:=\frac{2 r^{\frac{1}{2}\left(2-\alpha_{0}-\alpha_{u}\right)}}{a_{0}+a_{u}-2}=\frac{r^{-\kappa}}{\kappa}, \quad \kappa:=\frac{a_{0}+a_{u}}{2}-1, \quad v:=\frac{a_{0}+2 a_{1}+a_{u}-2}{2\left(a_{0}+a_{u}-2\right)}
$$

and $J_{v}(\tilde{r}), Y_{v}(\tilde{r})$ are the Bessel functions of first and second kind. The integration constants are found by looking at the canonical computation relations for theories in curved space-times and at the Neumann boundary condition to obtain (Figure 1)

$$
A_{\omega}=\sqrt{\frac{\pi \alpha^{\prime}}{|\kappa|\left(1+B_{\omega}^{2}\right)}}, \quad B_{\omega}=-\frac{J_{v-1}\left(\omega \tilde{r}_{b}\right)}{Y_{v-1}\left(\omega \tilde{r}_{b}\right)}
$$

where in the above and following computations we need to use the Bessel function properties presented in the Appendix of [14].

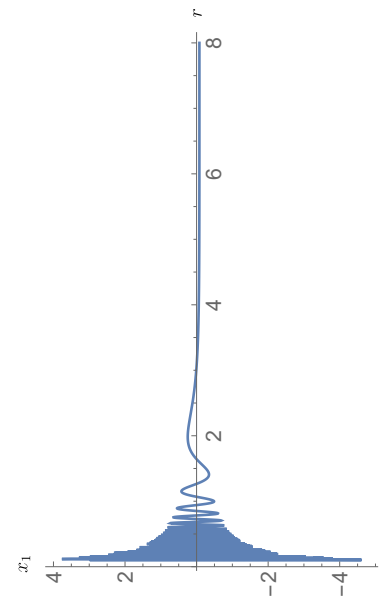

Figure 1: The real part of the string world-sheet fluctuations in a fixed Lifshitz background for a particular frequency and time. The large density of excitations in the deep IR $r$, get quickly smoothed out to result the corresponding Brownian motion on the boundary at large $r$.

The two-point function of the fluctuations in terms of the energy of the string in the low frequency limit

$$
\omega \ll \tilde{r}_{b}=\frac{r_{b}^{-\kappa}}{\kappa},
$$


takes a very compact form

$$
\left\langle X_{1}(t) X_{1}(0)\right\rangle \sim E^{\frac{4 k(1-v)}{\left(a_{0}-\kappa\right)}}|t|^{3-2 v}, \quad \text { when } \quad v \geq 1,
$$

and

$$
\left\langle X_{1}(t) X_{1}(0)\right\rangle \sim E^{0}|t|^{2 v-1}, \quad \text { when } \quad v \leq 1
$$

where $X_{1}(t):=\delta x_{1}\left(t, r_{b}\right)$. The energy of the string comes by trading the cut-off $r_{b}$ at the boundary using the action of the static straight string

$$
E=\frac{1}{2 \pi \alpha^{\prime}} \int_{r_{h}}^{r_{b}} d r \sqrt{-g_{00} g_{r r}}=\frac{1}{2 \pi \alpha^{\prime}}\left(a_{0}-\kappa\right) r_{b}^{a_{0}-\kappa} .
$$

To summarise the analysis, we find that after an involved computation the two-point function (2.12), (2.13) turns out to depend only on a single parameter, the order of the Bessel function. This is surprising and elegant result. We mention that in the unrelated studies of the chaos in the nonrelativistic theories, it has been found that the order of the Bessel function of closed string solutions controls whether the theory is chaotic or not [50]. This is another example where the order of Bessel function plays such a significant role.

Depending on the properties of the dual field theory of (2.6), the two-point function of the particle fluctuations is characterized by one of the two branches we derived, where one of them is always independent of the mass of the quark. The null energy condition (NEC) is satisfied for regions in both branches of the two-point function (2.12) and (2.13), ensuring that both of them can be physical. For theories giving $v=3 / 2$ and $v=1 / 2$, where the AdS background belongs, we have a minimal rate of logarithmic growth, while for theories giving $v=1$ we have the maximum rate of growth.

\subsubsection{Linear Response Function Analysis and Fluctuation-Dissipation Relation}

The response function of the system due to an applied external force $F(t)=E e^{-i \omega t} F(\omega)$ can be found from

$$
\left\langle X_{1}(\omega)\right\rangle=\chi(\omega) F(\omega)
$$

where $\chi(\omega)$ is the admittance of the system. The force corresponds to a new boundary term

$$
\frac{g_{11} \sqrt{-g_{00}}}{\sqrt{g_{u u}}} \delta x_{1}^{\prime}\left(r_{b}\right)=2 \pi \alpha^{\prime} F(t)
$$

which does not modify the solution of string fluctuations, only its constants. Making a tortoise coordinate transformation $r \rightarrow \tilde{r}$ we see that the fluctuation dynamics in the IR limit $r \rightarrow 0 \Rightarrow \tilde{r} \rightarrow \infty$ for $\kappa>0$, behaves like a wave equation in the flat space, where by choosing the ingoing boundary conditions we get

$$
\delta x_{1}(t, r)=e^{-i \omega t} g_{\omega}(r), \quad g_{\omega}(r)=r^{-v \kappa} H_{v}(\omega \tilde{r})
$$


with $H:=H^{(1)}=J+i Y$ being the Hankel function. Therefore the response function can be found to be

$$
\chi(\omega)=\frac{2 \pi \alpha^{\prime}}{\omega} r_{b}^{-a_{1}} \frac{H_{v}\left(\omega \tilde{r}_{b}\right)}{H_{v-1}\left(\omega \tilde{r}_{b}\right)} .
$$

All the information of the system and the theory are incorporated in the response function in an elegant way through the definition of $v$ and $\tilde{r}$. The fluctuation-dissipation theorem can be also shown that it is satisfied

$$
\operatorname{Im} \chi(\omega)=\frac{4 \alpha^{\prime}}{\omega^{2}} \frac{r_{b}^{-a_{1}}}{\tilde{r}_{b}}\left(J_{v-1}^{2}\left(\omega \tilde{r}_{b}\right)-Y_{v-1}^{2}\left(\omega \tilde{r}_{b}\right)\right)^{-1}=\frac{1}{2}\left\langle X_{1}(\omega) X_{1}(0)\right\rangle
$$

for the theories fitting in our study.

By expanding the response function in the positive $v$ region

$$
\chi(\omega) \sim-c_{1}\left[m(i \omega)^{2}+\mathscr{O}\left(\omega^{4}\right)+\gamma(-i \omega)^{2 v}+\mathscr{O}\left(\omega^{2 v+2}\right)\right]^{-1},
$$

we obtain the inertial mass $m$ and the self-energy $\gamma$ of the particle

$$
m=\frac{r_{b}^{2 \kappa(v-1)}}{2 \kappa(v-1)}, \quad \gamma=\frac{1-i \tan \left[\left(v-\frac{1}{2}\right) \pi\right]}{\left((2 i \kappa)^{2 v-1} \Gamma(v)^{2}\right)} \pi
$$

to find that the mass is not simply equal to the energy of the string given by (2.14). The order of the Bessel function plays a significant role in the result, indicating for example which term dominates in the expression. For $v>1$ the inertial mass dominates over the self energy at low frequency, otherwise the self energy is the dominant contribution.

\subsection{Thermal Diffusion of a Heavy Quark}

Let us now consider the Brownian motion in a thermal environment. The metric (2.6) has to contain a black hole

$$
g_{00}=r^{a_{0}} f(r), \quad g_{r r}=\frac{1}{r^{a_{u}} f(r)}, \quad g_{i i}=r^{a_{i}}, \quad f(r):=1-\frac{r_{h}^{a_{f}}}{r^{a_{f}}}, \quad r_{h} \neq 0, \quad T=\frac{a_{f}}{4 \pi} r_{h}^{\kappa}
$$

where $a_{f}$ is a constant and $T$ is the temperature of the heat bath. The form of the blackening factor does not need to be necessarily chosen as the function above, for most of our analysis would be enough to assume a single zero at the position of the horizon $r_{h}$.

The equations of motion for the thermal fluctuations are generated by (2.5) and can be brought to the Schrödinger-like form by a coordinate transformation presented in Appendix of [14] to get

$$
\frac{\partial^{2} y}{\partial r_{\star}^{2}}+\left(\omega^{2}-V(r)\right) y=0
$$

where

$$
\begin{aligned}
& y=h_{\omega}(r) r^{\frac{a_{1}}{2}}, \quad r_{\star}=-\frac{r_{h}^{-\kappa}}{a_{f}} B_{\left(\frac{r_{h}}{r}\right)^{a_{f}}}\left[\frac{\kappa}{a_{f}}, 0\right], \\
& V(r)=\frac{a_{1}}{2} r^{2 \kappa} f(r)\left(\left(a_{0}+a_{u}+\frac{a_{1}}{2}-1\right) f(r)+r f^{\prime}(r)\right),
\end{aligned}
$$


where $B_{z}[a, b]$ is the incomplete beta function. The monodromy patching procedure can be applied to obtain an approximate solution patching the regions between the black hole horizon all the way towards the boundary $[51,52,53,15,19]$. The three regions are chosen as

$$
\begin{array}{ll}
\text { A) } & r \sim r_{h} \quad \text { and } \quad V(r) \ll \omega^{2} \Longleftrightarrow f(r) \ll \omega . \\
\text { B) } & V(r) \gg \omega^{2} \Longleftrightarrow f(r) \gg \omega . \\
\text { C) } & r \rightarrow \infty \Longleftrightarrow r \gg r_{h} .
\end{array}
$$

giving the solutions for the Fourier modes $\delta x_{1}(t, r)=e^{-i \omega t} h_{\omega}(r)$ as [14]

$$
\begin{aligned}
& h_{A h}(r)=c_{1}\left(1-\frac{i \omega r_{h}^{-\kappa}}{a_{f}} \log \left(\frac{r}{r_{h}}-1\right)\right), \\
& h_{B h}(r)=c_{3}+c_{4} c_{0}+\frac{c_{4}}{a_{f}} r_{h}^{-2 \kappa v} \log \left(\frac{r}{r_{h}}-1\right), \\
& h_{B b}(r)=c_{3}-\frac{c_{4}}{2 \kappa v} r^{-2 \kappa v}, \\
& h_{C b}(r)=c_{5}+c_{6} r^{-2 \kappa v}\left(\frac{\omega \operatorname{sign}(1+2 \kappa)}{2 \kappa}\right)^{2 v},
\end{aligned}
$$

where the notation of the subscript used to identify which region of the space the solution covers: three regions and the proximity close to (h)orizon or (b)oundary is labeled. By patching the solutions we eventually obtain for their constants

$$
c_{5}=c_{1}\left(1+i c_{0} \omega r_{h}^{a_{1}}\right), \quad c_{6}=\frac{i \omega r_{h}^{a_{1}} c_{1}}{2 \kappa v}\left(\frac{2 \kappa}{\omega \operatorname{sign}(1+2 \kappa)}\right)^{2 v},
$$

to give the diffusive nature of the solution near the boundary

$$
h_{\omega}(r)=c_{1}\left(1+i \omega c_{0} r_{h}^{a_{1}}+\frac{i \omega r_{h}^{a_{1}}}{2 \kappa v} r^{-2 \kappa v}\right)
$$

\subsubsection{Response Function Analysis, Fluctuation-Dissipation and Nature of the Thermal Noise}

The response function expansion for low frequency is given by [14]

$$
\chi(\omega)=2 \pi \alpha^{\prime}\left(\frac{i}{\gamma \omega}-\frac{m}{\gamma^{2}}+\mathscr{O}(\omega)\right)
$$

where the damping coefficient and inertial mass reads

$$
\gamma=r_{h}^{a_{1}}, \quad m=r_{h}^{2 a_{1}}\left(-c_{0}+\frac{r_{b}^{-2 \kappa v}}{2 \kappa v}\right)+m_{0} .
$$

The mass receives a thermal correction compared with the zero temperature result $m_{0}(2.21)$. The diffusion constant in terms of the response function reads

$$
D=T \lim _{\omega \rightarrow 0}(-i \omega \chi(\omega))
$$


and is found to depend on powers of temperature specified by the order of the Bessel function [14]

$$
D=2 \pi \alpha^{\prime}\left(\frac{4 \pi}{a_{f}}\right)^{2 v-1} T^{2(1-v)} .
$$

The monotonicity of the coefficient with respect to temperature, is increasing for $v<1$ and decreasing for $v>1$, depending therefore on the characteristics of the dual field theory.

An interesting observation at the low frequency limit, is that the response function takes a universal form depending on the metric element along the spatial direction that the fluctuations occur and the temperature of the heat bath, given by the simple formula [14]

$$
\chi(\omega)=\frac{2 \pi \alpha^{\prime}}{-i \omega g_{11}\left(r_{h}\right)} .
$$

The fluctuation-dissipation theorem is found to hold when the density of states is $\sim \log \varepsilon /\left(4 \pi^{2} T\right)$. Moreover, it can be seen that the correlator of the random force $\xi$ is independent of the frequency $\omega$ indicating a white noise which depends on the temperature with powers of the Bessel function $\operatorname{order}\langle\xi \xi\rangle \sim T^{2 v}$.

\subsection{Dp-brane Fluctuations in a $d+1$-spacetime}

In [54] it has been noticed that the dynamics of the $k$-strings corresponding holographically to certain Dp-branes, can be mapped to the dynamics of fundamental strings in theories that have a certain preserved quantities under a T-duality. Here we consider another type of special branes, the rigid ones, and study the fluctuation and dissipation of the branes.

The brane is parametrized in the radial gauge as: $x_{0}=\tau, r=\sigma_{1}:=\sigma, x_{2}=\sigma_{2}, \ldots, x_{p}=$ $\sigma_{p}, x_{j}=x_{j}\left(\tau, \sigma_{i}\right), j=1, p+1, \ldots, d$. The fluctuations along the spatial transverse direction $x_{1}$ are given by the perturbation of the Dirac-Born-Infeld (DBI) action as [14]

$$
S_{\mathrm{DBI}, 2}=\frac{T_{p}}{2} \int d \tau d \sigma_{i}\left(-\frac{g_{11} \sqrt{-g_{00}} \sqrt{g_{22} \ldots g_{p p}}}{\sqrt{g_{r r}}} \delta x_{1}^{\prime 2}+\frac{g_{11} \sqrt{g_{r r}} \sqrt{g_{22} \ldots g_{p p}}}{\sqrt{-g_{00}}} \delta \dot{x}_{1}^{2}\right) .
$$

giving the mode equation for $\delta x_{1}(\tau, \sigma)=e^{-i \omega \tau} h_{\omega}(r)$

$$
\partial_{r}\left(-\frac{g_{11} \sqrt{-g_{00}} \sqrt{g_{22} \ldots g_{p p}}}{\sqrt{g_{r r}}} h_{\omega}(r)^{\prime}\right)-\frac{g_{11} \sqrt{g_{r r}} \sqrt{g_{22} \ldots g_{p p}}}{\sqrt{-g_{00}}} \omega^{2} h_{\omega}(r)=0 .
$$

We observe that under a mapping of the form [14]

$$
g_{11} \longrightarrow g_{11} \sqrt{g_{22} g_{33} \ldots g_{d d}} \text {, or equivalently } a_{1} \longrightarrow \tilde{a}_{1}=a_{1}+\frac{1}{2}\left(a_{2}+\ldots+a_{p}\right),
$$

the string fluctuation equations (2.5) become equivalent to the brane fluctuation equations. By defining the shifted constants

$$
\tilde{r}:=\frac{2 r^{\frac{1}{2}\left(2-\alpha_{0}-\alpha_{u}\right)}}{a_{0}+a_{u}-2}=\frac{r^{-\kappa}}{\kappa}, \quad \kappa:=\frac{a_{0}+a_{u}}{2}-1, \quad \tilde{v}:=\frac{a_{0}+2 \tilde{a}_{1}+a_{u}-2}{2\left(a_{0}+a_{u}-2\right)},
$$

the brane-related analysis follows in a straightforward way. For example the two-point function reads

$$
\left\langle X_{1}(t) X_{1}(0)\right\rangle \sim E^{\frac{4 \kappa(1-\tilde{v})}{\left(a_{0}-\kappa\right)}}|t|^{3-2 \tilde{v}}, \quad \text { when } \quad \tilde{v} \geq 1
$$


and

$$
\left\langle X_{1}(t) X_{1}(0)\right\rangle \sim E^{0}|t|^{2 \tilde{v}-1}, \quad \text { when } \quad \tilde{v} \leq 1 .
$$

The response function of the quantum brane fluctuations is

$$
\chi(\omega)=\frac{2 \pi \alpha^{\prime}}{\omega} r_{b}^{-a_{1}-\frac{1}{2}\left(a_{2}+\ldots+a_{p}\right)} \frac{H_{\tilde{v}}\left(\omega \tilde{r}_{b}\right)}{H_{\tilde{v}-1}\left(\omega \tilde{r}_{b}\right)} .
$$

with a low-frequency expansion that provides the inertial mass $m$ and the self-energy $\gamma$ of the state

$$
m=\frac{r_{b}^{2 \kappa(\tilde{v}-1)}}{2 \kappa(\tilde{v}-1)}, \quad \gamma=\frac{1-i \tan \left[\left(\tilde{v}-\frac{1}{2}\right) \pi\right]}{\left((2 i \kappa)^{2 \tilde{v}-1} \Gamma(\tilde{v})^{2}\right)} \pi .
$$

The analysis of the thermal fluctuations goes along the same lines with the string's one. For example for the case of branes the diffusion constant in terms of the temperature reads

$$
D=2 \pi \alpha^{\prime}\left(\frac{4 \pi}{a_{f}}\right)^{2 \tilde{v}-1} T^{2(1-\tilde{v})},
$$

where for $\tilde{v}<1$ increases with temperature and otherwise decreases.

For the branes in an arbitrary background the response function was proposed to take the form [14]

$$
\chi(\omega)=\frac{2 \pi \alpha^{\prime}}{-i \omega g_{11}\left(r_{h}\right) \sqrt{g_{22}\left(r_{h}\right) \ldots g_{p p}\left(r_{h}\right)}} .
$$

\subsection{Application of the Generic Formalism to Particular Theory}

Let us briefly demonstrate how the methodology described, applies to theories dual to the anisotropic black hole background found recently in Einstein-Axion-Dilaton action and contain as particular case the IIB supergravity background [46]. The geometry which accommodates a black hole, written in the form of $[46,14]$ reads

$$
d s^{2}=a^{2} C_{R} e^{\frac{\phi(r)}{2}} r^{-\frac{2 \theta}{d z}}\left(r^{2}\left(-f(r) d t^{2}+d x_{i}^{2}\right)+C_{Z} r^{\frac{2}{z}} d x_{3}^{2}+\frac{d r^{2}}{f(r) a^{2} r^{2}}\right),
$$

where

$$
f(r)=1-\left(\frac{r_{h}}{r}\right)^{d+(1-\theta) / z}, \quad e^{\frac{\phi(r)}{2}}=r^{\frac{\sqrt{\theta^{2}+3 z(1-\theta)-3}}{\sqrt{6 z}}}
$$

and

$$
C_{R}=\frac{(3 z-\theta)(1+3 z-\theta)}{6 z^{2}}, \quad C_{Z}=\frac{z^{2}}{2(z-1)(1+3 z-\theta)} .
$$

The background becomes of IIB supergravity solution for $z=3 / 2, \theta=0$ reproducing the geometry [43] and the IR geometry of the RG flow [44]. The Hawking temperature of the theory can be found equal to

$$
T=\frac{|d+(1-\theta) / z|}{4 \pi r_{h}^{z}}
$$


Let us work with $d=3$ spatial dimensions. The first task is to determine the order of the Bessel functions for fluctuations along each direction by using (2.9)

$$
\begin{aligned}
& v_{1}=\frac{18 z-4 \theta+\sqrt{6} \sqrt{3 z(1-\theta)-3+\theta^{2}}}{12 z}, \\
& v_{3}=\frac{12+6 z-4 \theta+\sqrt{6} \sqrt{3 z(1-\theta)-3+\theta^{2}}}{12 z} .
\end{aligned}
$$

The fluctuations along the $x_{1}$ and $x_{3}$ behave in a different manner. The two-point function along $x_{1}$ is

$$
\left\langle X_{1}(t) X_{1}(0)\right\rangle \sim E^{-2}|t|^{3-2 v_{1}}, \quad \text { when } \quad v_{1} \geq 1
$$

where for $v_{1}<1$ there is no physical and stable theory. The $x_{3}$ fluctuations give

$$
\left\langle X_{3}(t) X_{3}(0)\right\rangle \sim E^{2 \frac{12-6 z-4 \theta+\sqrt{6} \sqrt{3 z(1-\theta)-3+\theta^{2}}}{-6 z+4 \theta-\sqrt{6} \sqrt{3 z(1-\theta)-3+\theta^{2}}}}|t|^{3-2 v_{3}}, \quad \text { when } \quad v_{3} \geq 1
$$

or

$$
\left\langle X_{3}(t) X_{3}(0)\right\rangle \sim E^{0}|t|^{2 v_{3}-1}, \quad \text { when } \quad v_{3} \leq 1 .
$$

The diffusion constant depends on the direction and is given by

$$
D_{i}=2 \pi \alpha^{\prime}\left(\frac{4 \pi}{d+(1 \theta) / z}\right)^{\left(2 v_{i}-1\right)} T^{2\left(1-v_{i}\right)}
$$

where $i=1,3$ labels the direction of the fluctuations.

\section{Stochastic Motion of the Moving Heavy Quark: Theory Independent Approach}

Let us consider a heavy particle moving with a velocity $v$ in a strongly coupled environment. The Langevin coefficients $\kappa_{\perp}, \kappa_{\|}$, corresponding to the mean squared momentum per unit of time in transverse and parallel direction with respect to the quark's motion, are obtained by analyzing the fluctuation of the trailing Wilson line. The out of equilibrium relativistic heavy quarks go under a Brownian motion with a stochastic force $\xi(t)$. The methodology for generic theories including the anisotropic ones, was developed in $[55,56]$ by providing a set of readily applicable formulas for the observables and extracting certain behaviors of them in a wide class of theories. The set of theories accommodated in this study include for example: conformal, non-relativistic, hyperscaling violation, anisotropic and theories under certain magnetic fields. The formulas of [55, 56] have been applied on several particular models, for example in [57]. The initiating works on the subject include $[58,59,60,61,62]$ and early works that contributed to further development include [63, 64, 65, 66].

It is worthy to mention, that by studying the relativistic heavy quark diffusion in theories with rotational invariance it has been found that a universal inequality for the Langevin coefficients exist $\kappa_{\|} \geq \kappa_{\perp}[55,66]$. Namely the longitudinal Langevin diffusion coefficient along the quark motion is larger compared to that of the transverse direction. This inequality has been proved to be 
violated in the presence of strongly coupled anisotropies [55, 56], in a similar way with the well known shear viscosity over entropy density bound $[67,68,69,46]$ (other relevant works include $[70,71,72,73])$.

Other related works on the drag and stochastic sting motion include $[74,75,63,76,77,78,79$, $80,81,82,83,84,85,86]$ while dragging of the particle even at zero temperature in non-relativistic theories has been observed [87, 88, 89]. An earlier review with references therein is [90].

\subsection{The Trailing String}

Let us review first the theory-independent analysis of the trailing string following for the drag force analysis of the Appendices [91, 92] while for the stochastic motion analysis we follow [55, 56]. We use a similar metric to (2.1) with $u=1 / r$ and therefore a boundary at $u \rightarrow 0$ and a black hole horizon at $u=u_{h}$

$$
d s^{2}=-g_{00}(u) d x_{0}^{2}+g_{u u}(u) d u^{2}+\sum_{i=1}^{d} g_{i i}(u) d x_{i}^{2} .
$$

The string parametrization for a quark moving on the $x_{1}$ direction with velocity $v$, is $x_{0}=\tau, u=$ $\sigma, x_{1}=v t+\xi(u)$, where $\xi$ is the profile of the string in the bulk, satisfying on the boundary $\xi\left(u_{b}\right)=0$. The equation of motion is

$$
\xi^{\prime 2}=-g_{\text {uи }} C^{2} \frac{g_{00}+g_{11} v^{2}}{g_{00} g_{11}\left(C^{2}+g_{00} g_{11}\right)}, \quad C:=2 \pi \alpha^{\prime} \Pi_{u}^{1},
$$

where $u_{0}$ is the black hole horizon of the world-sheet metric given by the solution of

$$
g_{00}\left(u_{0}\right)=-g_{11}\left(u_{0}\right) v^{2}
$$

when $g_{\text {uu }}\left(u_{0}\right) \neq 0$. For $v=0$ the horizon of the worldsheet coincides with the horizon of the black hole, satisfying the natural expectations. The dragging of the particle is given by [91]

$$
F_{\text {drag }, x_{1}}=-\frac{1}{2 \pi \alpha^{\prime}} \frac{\sqrt{-g_{00}\left(u_{0}\right) g_{11}\left(u_{0}\right)}}{2 \pi}=-\frac{g_{11}\left(u_{0}\right) v}{2 \pi \alpha^{\prime}},
$$

while the friction coefficient is defined by

$$
F_{\text {drag }}=\frac{d p}{d t}=-\eta_{D} p, \quad \eta_{D}=\frac{g_{11}\left(u_{0}\right)}{2 \pi \alpha^{\prime} M \gamma},
$$

where $M$ is the mass of the heavy quark, $p=M v \gamma$ and $\gamma$ is the Lorentz factor. The worldsheet has a blackening factor, and therefore a temperature is associated to it. To find the temperature we diagonalize the world-sheet metric to get [55]

$$
T_{w s}^{2}=\left.\frac{1}{16 \pi^{2}}\left|\frac{1}{g_{00} g_{u u}}\left(g_{00} g_{11}\right)^{\prime}\left(\frac{g_{00}}{g_{11}}\right)^{\prime}\right|\right|_{u=u_{0}} .
$$

$T_{w s}$ should be thought as the effective temperature that the quark feels and is the temperature that appears in the Einstein equations relating the diffusion and the Langevin coefficients. The effective temperature in most theories turns out to be lower than the heat bath temperature, although in anisotropic theories may become higher. The natural expectation for the static quark $(v=0)$ would be that it feels the heat bath temperature and this can be verified by the above relation to obtain $T_{w s}=T$. 


\subsection{Fluctuation of the Moving Trailing String}

Let us review the fluctuations around the trailing string in a generic background. The method was developed in $[55,56]$ and the action was found to be

$$
S_{2}=-\frac{1}{2 \pi \alpha^{\prime}} \int d \tau d \sigma \frac{H^{\alpha \beta}}{2}\left[N(u) \partial_{\alpha} \delta x_{1} \partial_{\beta} \delta x_{1}+\sum_{i \neq 1} g_{i i} \partial_{\alpha} \delta x_{i} \partial_{\beta} \delta x_{i}\right],
$$

where $H^{\alpha \beta}=\sqrt{-h} h^{\alpha \beta}$, and $h^{\alpha \beta}$ is inverse of the diagonalized induced world-sheet metric given by

$$
h_{\sigma \sigma}=\frac{g_{00} g_{u u} g_{11}}{g_{00} g_{11}+C^{2}}, \quad h_{\tau \tau}=g_{00}+v^{2} g_{11}
$$

Taking advantage of the membrane paradigm it has been found that the Langevin coefficients are computed by $[55,56]$

$$
\kappa_{\perp}=\left.\frac{1}{\pi \alpha^{\prime}} g_{k k}\right|_{u=u_{0}} T_{w s}, \quad \kappa_{\|}=\left.\frac{1}{\pi \alpha^{\prime}} \frac{\left(g_{00} g_{11}\right)^{\prime}}{g_{11}\left(\frac{g_{00}}{g_{11}}\right)^{\prime}}\right|_{u=u_{0}} T_{w s},
$$

where the index $k$ denotes a particular transverse direction to that of motion $x_{1}$ and no summation is taken. The ratio takes the surprisingly compact form

$$
\frac{\kappa_{\|}}{\kappa_{\perp}}=\left.\frac{\left(g_{00} g_{11}\right)^{\prime}}{g_{k k} g_{11}\left(\frac{g_{00}}{g_{11}}\right)^{\prime}}\right|_{u=u_{0}} .
$$

In isotropic spaces it has been found that $\kappa_{\|}>\kappa_{\perp}$ for any velocity of the quark's motion [55, 66]. In anisotropic theories the universal condition is violated [55, 56]. It exists a critical quark velocity $v_{c}$ beyond which the inequality gets inverted to $\kappa_{\|}<\kappa_{\perp}$.

\subsection{Excitation of the Medium due to Heavy Quark Motion}

The quark deposits energy in the medium through its interactions with the environment, and as a result excitations in the medium occur which are expected to be well described by linearized hydrodynamics. Due to the motion of the particle through the plasma, a laminar wake is generated behind it, which has been shown to be of universal strength with respect to the total drag force exerted by the plasma. A sonic boom has been discovered for probes moving faster than the speed of sound and a diffusion wake behind the quark's motion has been also found [93, 94, 95, 96].

The total action to compute such backreacted effects is given by

$$
S=\frac{1}{2 \kappa^{2}} \int d^{5} x \sqrt{-g}\left(R+\frac{12}{L^{2}}\right)-S_{N G},
$$

where the NG is computed for the trailing string of the section 3.1. The equations of motion read

$$
R_{\mu \nu}-\frac{1}{2} g_{\mu v} R-\frac{6}{L^{2}} g_{\mu v}=\tau_{\mu v} .
$$


If we concentrate on the AdS spacetime

$$
d s^{2}=\frac{1}{u^{2}}\left(-f(u) d t^{2}+d \vec{x}^{2}+\frac{d u^{2}}{f(u)}\right), \quad f(u)=1-\frac{u^{4}}{u_{h}^{4}},
$$

the bulk stress-energy tensor for the trailing string takes the form

$$
\tau_{\mu v}=-\frac{\kappa^{2}}{2 \pi \alpha^{\prime}} u^{3} \sqrt{1-v^{2}} \partial_{\alpha} x^{\mu} \partial^{a} x^{v}
$$

computed on the string world-sheet. The backreaction of the string metric can be found by considering the fluctuations $g_{\mu v}=g_{\mu \nu}^{0}+h_{\mu \nu}$ on the $g_{\mu \nu}^{0}$ AdS metric. The $h_{\mu v}$ depends for the trailing string in terms of $x_{1}-v t$ and we have the freedom to take the axial gauge $h_{\mu u}=0$. The system of equations consists of ten second order differential equations in $u$, minus five first order constraints, therefore we need to specify fifteen integration constants. These are fixed by imposing conditions on the boundary of the space and at the horizon of the black hole as in [97].

The low momentum asymptotics can be obtained analytically by formally expanding all variables in low momenta, where the diffusion pole and the sound pole expected from the hydrodynamic behavior of the plasma are confirmed by the computations [97]. On the other hand at the large momenta, the leading term of the stress-energy tensor is a boosted version of the stationary quark's as expected. At scales much shorter that the typical length scale of the fluid the quark does not see the plasma and feels a vacuum state. The next order reveals the existence of a critical velocity for the quark's motion that passes from a region of energy depletion behind the quark, to a region of energy depletion in front of it. The full numerical analysis is presented in [97] for the various quark velocities.

\subsection{Non-Perturbative Monte Carlo Simulations of the Heavy Quark Diffusion}

An estimate of the heavy quark momentum diffusion with Monte-Carlo simulation using the Backus-Gilbert method [98] was done in [99] giving

$$
\kappa=1.8 \ldots 3.4 T^{3} .
$$

The result seems to be in agreement with a next to leading order computation in perturbative QCD using the hard thermal loop effective theory and by setting the scales and the coupling to the usual ones used in the heavy ion collisions [100]. Then the diffusion coefficient is estimated to

$$
D T=0.35 \ldots 1.1
$$

and has higher values compared to the one predicted for the light quarks in the continuum limit [101, 102]. This can be naturally explained with the fact that the heavy quarks feel slightly weaker interactions. Using these methods, it would be interesting to show that the heavy mass limit is justified for the lighter charm quarks, and not only for the heavier bottoms quarks. A promising direction would also be to estimate the effects from dynamical quarks on the heavy quark diffusion, where the screening should affect the observables. To this direction the gauge/gravity duality could also provide very insightful qualitative results. In this context, the flavor is added to the correspondence with the use of the Dp-branes, where the quenched limit is easier tractable [103, 104], while the unquenched limit is demanding computationally $[105,106]$, so approximate or numerical methods have been developed and the relevant screening on several observables has been observed, for example [107, 108, 109, 110, 111, 112]. 


\section{Summary}

In this brief review we have presented a theory independent treatment of stochastic heavy quark dynamics. In the introduction we have justified why the heavy quarks admit a stochastic treatment. Then we have presented the analysis of quantum and thermal fluctuations for a static quark by considering fluctuations of the straight string. We have moved on to the analysis of the trailing string fluctuations to obtain the Langevin equations. The idea of this review is to present the model independent holographic formulas applicable to wide class of theories, which have been obtained in [14] for the static quark and in $[55,56]$ for the trailing string. We briefly summarize most of the formulas below.

Quantum Fluctuations of the Heavy Particle: Using the wide class of theories described by (2.1) we obtain the generic form of the action (2.3) describing the fluctuations with the mode equation (2.5). For the theories of the form (2.6) the fluctuations are given by a Bessel type solution (2.8) with order $v$ (2.9) which depends on the background geometry. By applying the boundary conditions and appropriate quantization we determine the constants of the solutions. The two pointfunction of the fluctuation has a surprisingly compact form and the two branches (2.12) and (2.13), controlled by the value of the Bessel function and therefore by the properties of the theory we study.

The response function analysis is done by applying the generic boundary force (2.16). The modification of the boundary condition leads to a different solution for the fluctuations specified by (2.17). The response function is found in terms of the Hankel function with the same order $v$. Then the fluctuation-dissipation theorem is found to be satisfied (2.19). By expanding the response function we determine the inertial mass and the self energy of the particle for the whole class of theories (2.21). All the results and their properties heavily depend on the order $v$ incorporating the information of the theory.

Thermal Diffusion of the Heavy Particle: Including to our geometry the black hole (2.22), we study the thermal string fluctuations. The solution to the equation of fluctuations (2.23) is involved and the monodromy patching method needs to be used, patching certain approximate solutions in different regions along the holographic direction (2.29). The solution close to the boundary is given by (2.31) depending heavily of the asymptotics of the metric element along the fluctuations and the Bessel function order. The response function is found to take the form (2.36), exclusively depending on the black hole horizon along the direction of fluctuations. The self energy and the thermally corrected inertial mass are given by (2.33). Interestingly the diffusion coefficient scales with the temperature in way that is solely controlled by the Bessel function order (2.35), realizing how elegantly the information of the wide class backgrounds is encoded in the order $v$.

Diffusion of a Rigid type Dp-Branes: By mapping the equations of the rigid branes (2.38) to the string fluctuations we find a way to read all the stochastic observables and coefficients from the analysis done on the strings. The mapping of the string to brane fluctuation is implemented by (2.39) giving the shifted Bessel function order (2.40). Then the two-point function of Dpbranes (2.41), (2.42), the response function (2.46), the inertial mass and self energy (2.44), and the diffusion coefficient (2.45) can be read in a straightforward way from the prescription explained above.

Dragging of the Heavy Moving Particle: We consider a heavy particle moving with a ve- 
locity $v$ in a strongly coupled environment described by a metric with elements being arbitrary functions of the holographic direction (3.1). The profile of the trailing string is given by the equation (3.2) and the two-dimensional worldsheet has a black hole with a horizon given by the solution of the equation (3.3). The drag force is expressed in terms of the metric element of the direction of the quark's motion (3.4), while the friction coefficient is found in (3.5). The moving quark feels an effective temperature different than the one of the heat bath given by the equation (3.6).

Langevin Coefficients of the Heavy Moving Particle: The action of the fluctuations is given by (3.7). Employing the membrane paradigm we obtain the Langevin coefficients given in terms of the metric elements and the effective temperature that the quark feels (3.9). These are generic powerful formulas directly applicable to the thermal holographic theories. The ratio of the two coefficients take a very simple form given by (3.10) and turns out that for isotropic theories it satisfies a universal relation inequality of the form $\kappa_{\|}>\kappa_{\perp}$ for the whole range of the quark motion velocity. In anisotropic theories the universal inequality gets inverted for a critical speed of the quark, and the universality is violated in a similar way that the viscosity over entropy bound is violated in anisotropic theories.

\section{References}

[1] R. Brown and J. J. Bennett, The miscellaneous botanical works of Robert Brown., vol. 1. London,Published for the Ray society by R. Hardwicke,

[2] A. Einstein, Über die von der molekularkinetischen Theorie der Wärme geforderte Bewegung von in ruhenden Flüssigkeiten suspendierten Teilchen, Annalen der Physik 322 (1905) 549.

[3] W. Sutherland, Lxxv. a dynamical theory of diffusion for non-electrolytes and the molecular mass of albumin, The London, Edinburgh, and Dublin Philosophical Magazine and Journal of Science 9 (1905) 781 [https://doi.org/10.1080/14786440509463331].

[4] von Smoluchowski M., Zur kinetischen theorie der brownschen molekularbewegung und der suspensionen, Annalen der Physik 326756 [https://onlinelibrary.wiley.com/doi/pdf/10.1002/andp.19063261405].

[5] J. Perrin, Mouvement brownien et réalité moléculaire, Annales de Chimie et de Physique 18 (1909) 5.

[6] M. C. Wang and G. E. Uhlenbeck, On the theory of the brownian motion ii, Rev. Mod. Phys. 17 (1945) 323.

[7] P. Hanggi and F. Marchesoni, Introduction: 100years of brownian motion, Chaos: An Interdisciplinary Journal of Nonlinear Science 15 (2005) 026101

[https://doi.org/10.1063/1.1895505].

[8] J. Dunkel and P. Hanggi, Relativistic brownian motion, Physics Reports 471 (2009) 1.

[9] X. Bian, C. Kim and G. E. Karniadakis, 111 years of brownian motion, Soft Matter 12 (2016) 6331.

[10] B. Svetitsky, Diffusion of charmed quarks in the quark-gluon plasma, Phys. Rev. D37 (1988) 2484.

[11] H. van Hees and R. Rapp, Thermalization of heavy quarks in the quark-gluon plasma, Phys. Rev. C71 (2005) 034907 [nucl-th/ 0412015 ].

[12] G. D. Moore and D. Teaney, How much do heavy quarks thermalize in a heavy ion collision?, Phys. Rev. C71 (2005) 064904 [hep-ph/ 0412346$].$ 
[13] M. G. Mustafa, Energy loss of charm quarks in the quark-gluon plasma: Collisional versus radiative, Phys. Rev. C72 (2005) 014905 [hep-ph/ 0412402 ].

[14] D. Giataganas, D.-S. Lee and C.-P. Yeh, Quantum Fluctuation and Dissipation in Holographic Theories: A Unifying Study Scheme, 1802.04983.

[15] J. de Boer, V. E. Hubeny, M. Rangamani and M. Shigemori, Brownian motion in AdS/CFT, JHEP 0907 (2009) 094 [0812.5112].

[16] D. T. Son and D. Teaney, Thermal Noise and Stochastic Strings in AdS/CFT, JHEP 07 (2009) 021 [0901.2338].

[17] S. Caron-Huot, P. M. Chesler and D. Teaney, Fluctuation, dissipation, and thermalization in non-equilibrium AdS5 black hole geometries, Phys. Rev. D84 (2011) 026012 [1102.1073].

[18] J. Sonner and A. G. Green, Hawking Radiation and Non-equilibrium Quantum Critical Current Noise, Phys. Rev. Lett. 109 (2012) 091601 [1203.4908].

[19] D. Tong and K. Wong, Fluctuation and Dissipation at a Quantum Critical Point, Phys. Rev. Lett. 110 (2013) 061602 [1210 . 1580].

[20] V. E. Hubeny and M. Rangamani, A Holographic view on physics out of equilibrium, Adv. High Energy Phys. 2010 (2010) 297916 [1006.3675].

[21] W. Fischler, P. H. Nguyen, J. F. Pedraza and W. Tangarife, Fluctuation and dissipation in de Sitter space, JHEP 08 (2014) 028 [1404.0347].

[22] C.-P. Yeh, J.-T. Hsiang and D.-S. Lee, Holographic influence functional and its application to decoherence induced by quantum critical theories, Phys. Rev. D91 (2015) 046009 [1410.7111].

[23] C.-P. Yeh and D.-S. Lee, Subvacuum effects in quantum critical theories from a holographic approach, Phys. Rev. D93 (2016) 126006 [1510 . 05778].

[24] P. Banerjee, Holographic Brownian motion at finite density, Phys. Rev. D94 (2016) 126008 [1512.05853].

[25] R. W. Moerman and W. A. Horowitz, A semi-classical recipe for wobbly limp noodles in partonic soup, 1605.09285.

[26] D.-S. Lee and C.-P. Yeh, A holographic description of negative energy states, JHEP 09 (2016) 059 [1606.02420].

[27] E. Kiritsis, L. Mazzanti and F. Nitti, The confining trailing string, JHEP 02 (2014) 081 [1311.2611].

[28] S.-H. Ho, W. Li, F.-L. Lin and B. Ning, Quantum Decoherence with Holography, JHEP 01 (2014) 170 [1309.5855].

[29] W. Fischler, J. F. Pedraza and W. Tangarife Garcia, Holographic Brownian Motion in Magnetic Environments, JHEP 12 (2012) 002 [1209.1044].

[30] D. Roychowdhury, Quantum fluctuations and thermal dissipation in higher derivative gravity, Nucl. Phys. B897 (2015) 678 [1506.04548].

[31] L. D. Landau and E. M. Lifshitz, Statistical Physics, Part I, Pergamon Press, Oxford Course of Theoretical Physics, Vol. 5, Third Edition (1980).

[32] A. Caldeira and A. Leggett, Path integral approach to quantum brownian motion, Physica A: Statistical Mechanics and its Applications 121 (1983) 587. 
[33] J. Schwinger, Brownian motion of a quantum oscillator, Journal of Mathematical Physics 2 (1961) 407 [https://doi.org/10.1063/1.1703727].

[34] R. P. Feynman and F. L. Vernon, Jr., The Theory of a general quantum system interacting with a linear dissipative system, Annals Phys. 24 (1963) 118.

[35] H. Grabert, P. Schramm and G. L. Ingold, Quantum Brownian motion: The Functional inegral approach, Phys. Rept. 168 (1988) 115.

[36] B. L. Hu and A. Matacz, Quantum Brownian motion in a bath of parametric oscillators: A Model for system - field interactions, Phys. Rev. D49 (1994) 6612 [gr-qc/9312035].

[37] B. L. Hu and H. E. Kandrup, Entropy Generation in Cosmological Particle Creation and Interactions: A Statistical Subdynamics Analysis, Phys. Rev. D35 (1987) 1776.

[38] J.-T. Hsiang and D.-S. Lee, Influence on electron coherence from quantum electromagnetic fields in the presence of conducting plates, Phys. Rev. D73 (2006) 065022 [hep-th/ 0512059 ].

[39] J.-T. Hsiang, T.-H. Wu and D.-S. Lee, Stochastic Lorentz forces on a point charge moving near the conducting plate, Phys. Rev. D77 (2008) 105021 [0706.3075].

[40] S. Kachru, X. Liu and M. Mulligan, Gravity Duals of Lifshitz-like Fixed Points, Phys.Rev. D78 (2008) 106005 [0808.1725].

[41] X. Dong, S. Harrison, S. Kachru, G. Torroba and H. Wang, Aspects of holography for theories with hyperscaling violation, JHEP 1206 (2012) 041 [1201.1905].

[42] K. Narayan, On Lifshitz scaling and hyperscaling violation in string theory, Phys. Rev. D85 (2012) 106006 [1202.5935].

[43] T. Azeyanagi, W. Li and T. Takayanagi, On String Theory Duals of Lifshitz-like Fixed Points, JHEP 0906 (2009) 084 [0905.0688].

[44] D. Mateos and D. Trancanelli, The anisotropic $N=4$ super Yang-Mills plasma and its instabilities, Phys.Rev.Lett. 107 (2011) 101601 [1105.3472].

[45] D. Mateos and D. Trancanelli, Thermodynamics and Instabilities of a Strongly Coupled Anisotropic Plasma, JHEP 1107 (2011) 054 [1106.1637].

[46] D. Giataganas, U. Gursoy and J. F. Pedraza, Strongly-coupled anisotropic gauge theories and holography, 1708.05691.

[47] S. Jain, N. Kundu, K. Sen, A. Sinha and S. P. Trivedi, A Strongly Coupled Anisotropic Fluid From Dilaton Driven Holography, JHEP 01 (2015) 005 [1406.4874].

[48] A. Donos, J. P. Gauntlett and O. Sosa-Rodriguez, Anisotropic plasmas from axion and dilaton deformations, JHEP 11 (2016) 002 [1608 . 02 970].

[49] M. Edalati, J. F. Pedraza and W. Tangarife Garcia, Quantum Fluctuations in Holographic Theories with Hyperscaling Violation, Phys. Rev. D87 (2013) 046001 [1210 . 6993].

[50] D. Giataganas and K. Sfetsos, Non-integrability in non-relativistic theories, JHEP 06 (2014) 018 [1403.2703].

[51] L. Motl and A. Neitzke, Asymptotic black hole quasinormal frequencies, Adv. Theor. Math. Phys. 7 (2003) 307 [hep-th/0301173].

[52] J. M. Maldacena and A. Strominger, Black hole grey body factors and d-brane spectroscopy, Phys. Rev. D55 (1997) 861 [hep-th/9609026]. 
[53] T. Harmark, J. Natario and R. Schiappa, Greybody Factors for d-Dimensional Black Holes, Adv. Theor. Math. Phys. 14 (2010) 727 [0708.0017].

[54] D. Giataganas, k-Strings as Fundamental Strings, JHEP 05 (2015) 134 [1503 . 09180 ].

[55] D. Giataganas and H. Soltanpanahi, Universal Properties of the Langevin Diffusion Coefficients, Phys.Rev. D89 (2014) 026011 [1310 . 6725].

[56] D. Giataganas and H. Soltanpanahi, Heavy Quark Diffusion in Strongly Coupled Anisotropic Plasmas, JHEP 06 (2014) 047 [1312. 7474$].$

[57] S. I. Finazzo, R. Critelli, R. Rougemont and J. Noronha, Momentum transport in strongly coupled anisotropic plasmas in the presence of strong magnetic fields, Phys. Rev. D94 (2016) 054020 [1605.06061].

[58] C. Herzog, A. Karch, P. Kovtun, C. Kozcaz and L. Yaffe, Energy loss of a heavy quark moving through N=4 supersymmetric Yang-Mills plasma, JHEP 0607 (2006) 013 [hep-th/ 0605158 ].

[59] S. S. Gubser, Drag force in AdS/CFT, Phys.Rev. D74 (2006) 126005 [hep-th/ 0605182 ].

[60] S. S. Gubser, Momentum fluctuations of heavy quarks in the gauge-string duality, Nucl.Phys. B790 (2008) 175 [hep-th/0612143].

[61] J. Casalderrey-Solana and D. Teaney, Heavy quark diffusion in strongly coupled N=4 Yang-Mills, Phys.Rev. D74 (2006) 085012 [hep-ph/ 0605199 ].

[62] J. Casalderrey-Solana and D. Teaney, Transverse Momentum Broadening of a Fast Quark in a N=4 Yang Mills Plasma, JHEP 0704 (2007) 039 [hep-th/ 0701123 ].

[63] G. C. Giecold, E. Iancu and A. H. Mueller, Stochastic trailing string and Langevin dynamics from AdS/CFT, JHEP 07 (2009) 033 [0903.1840].

[64] U. Gursoy, E. Kiritsis, G. Michalogiorgakis and F. Nitti, Thermal Transport and Drag Force in Improved Holographic QCD, JHEP 0912 (2009) 056 [0 906.1890 ].

[65] C. Hoyos-Badajoz, Drag and jet quenching of heavy quarks in a strongly coupled $N=2 *$ plasma, JHEP 0909 (2009) 068 [0907 . 5036$].$

[66] U. Gursoy, E. Kiritsis, L. Mazzanti and F. Nitti, Langevin diffusion of heavy quarks in non-conformal holographic backgrounds, JHEP 1012 (2010) 088 [1 006 . 3261 ].

[67] P. Kovtun, D. T. Son and A. O. Starinets, Viscosity in strongly interacting quantum field theories from black hole physics, Phys. Rev. Lett. 94 (2005) 111601 [hep-th/ 0405231 ].

[68] A. Rebhan and D. Steineder, Violation of the Holographic Viscosity Bound in a Strongly Coupled Anisotropic Plasma, Phys. Rev. Lett. 108 (2012) 021601 [1110.6825].

[69] S. Jain, R. Samanta and S. P. Trivedi, The Shear Viscosity in Anisotropic Phases, JHEP 10 (2015) 028 [1506.01899].

[70] J. Erdmenger, P. Kerner and H. Zeller, Non-universal shear viscosity from Einstein gravity, Phys. Lett. B699 (2011) 301 [1011.5912].

[71] R. Samanta, R. Sharma and S. P. Trivedi, Shear viscosity in an anisotropic unitary Fermi gas, Phys. Rev. A96 (2017) 053601 [1607.04799].

[72] X.-H. Ge, Notes on shear viscosity bound violation in anisotropic models, Sci. China Phys. Mech. Astron. 59 (2016) 630401 [1510.06861]. 
[73] K. S. Kolekar, D. Mukherjee and K. Narayan, Hyperscaling violation and the shear diffusion constant, Phys. Lett. B760 (2016) 86 [1604. 05092].

[74] S. S. Gubser, Comparing the drag force on heavy quarks in N=4 super-Yang-Mills theory and QCD, Phys. Rev. D76 (2007) 126003 [hep-th/ 0611272$].$

[75] J. Casalderrey-Solana, K.-Y. Kim and D. Teaney, Stochastic String Motion Above and Below the World Sheet Horizon, JHEP 12 (2009) 066 [0908 . 1470 ].

[76] K. Rajagopal and A. V. Sadofyev, Chiral drag force, JHEP 10 (2015) 018 [1505. 07379 ].

[77] C. P. Herzog and A. Vuorinen, Spinning Dragging Strings, JHEP 10 (2007) 087 [0 708.0609 ].

[78] Y. Akamatsu, Langevin dynamics and decoherence of heavy quarks at high temperatures, Phys. Rev. C92 (2015) 044911 [1503.08110].

[79] W. A. Horowitz, Fluctuating heavy quark energy loss in a strongly coupled quark-gluon plasma, Phys. Rev. D91 (2015) 085019 [1501.04693].

[80] S. Nakamura and H. Ooguri, Out of Equilibrium Temperature from Holography, Phys. Rev. D88 (2013) 126003 [1309.4089].

[81] D. Arean, L. A. Pando Zayas, L. Patino and M. Villasante, Velocity Statistics in Holographic Fluids: Magnetized Quark-Gluon Plasma and Superfluid Flow, JHEP 10 (2016) 158 [1606.03068].

[82] S. Roy, Holography and drag force in thermal plasma of non-commutative Yang-Mills theories in diverse dimensions, Phys. Lett. B682 (2009) 93 [0907. 0333].

[83] W. A. Horowitz and Y. V. Kovchegov, Shock Treatment: Heavy Quark Drag in a Novel AdS Geometry, Phys. Lett. B680 (2009) 56 [0904.2536].

[84] E. Caceres and A. Guijosa, On Drag Forces and Jet Quenching in Strongly Coupled Plasmas, JHEP 12 (2006) 068 [hep-th/ 0606134$].$

[85] M. Ahmadvand and K. Bitaghsir Fadafan, Energy loss at zero temperature from extremal black holes, 1512.05290.

[86] Z.-q. Zhang, K. Ma and D.-f. Hou, Drag force in strongly coupled supersymmetric YangâĂŞMills plasma in a magnetic field, J. Phys. G45 (2018) 025003 [1802 . 01912].

[87] S. A. Hartnoll, J. Polchinski, E. Silverstein and D. Tong, Towards strange metallic holography, JHEP 04 (2010) 120 [0912. 1061$].$

[88] E. Kiritsis, Lorentz violation, Gravity, Dissipation and Holography, JHEP 01 (2013) 030 [1207.2325]

[89] K. B. Fadafan, Drag force in asymptotically Lifshitz spacetimes, 0912 . 4873.

[90] J. Casalderrey-Solana, H. Liu, D. Mateos, K. Rajagopal and U. A. Wiedemann, Gauge/String Duality, Hot QCD and Heavy Ion Collisions, 1101.0618.

[91] D. Giataganas, Probing strongly coupled anisotropic plasma, JHEP 1207 (2012) 031 [1202.4436].

[92] D. Giataganas, Observables in Strongly Coupled Anisotropic Theories, PoS Corfu2012 (2013) 122 [1306.1404].

[93] S. S. Gubser, S. S. Pufu and A. Yarom, Sonic booms and diffusion wakes generated by a heavy quark in thermal AdS/CFT, Phys. Rev. Lett. 100 (2008) 012301 [0706.4307]. 
[94] S. S. Gubser and A. Yarom, Universality of the diffusion wake in the gauge-string duality, Phys. Rev. D77 (2008) 066007 [0709.1089].

[95] J. Casalderrey-Solana, E. V. Shuryak and D. Teaney, Hydrodynamic flow from fast particles, hep-ph/0602183.

[96] J. J. Friess, S. S. Gubser, G. Michalogiorgakis and S. S. Pufu, The Stress tensor of a quark moving through N=4 thermal plasma, Phys. Rev. D75 (2007) 106003 [hep-th/ 0607022 ].

[97] S. S. Gubser, S. S. Pufu, F. D. Rocha and A. Yarom, Energy loss in a strongly coupled thermal medium and the gauge-string duality, in Quark-gluon plasma 4 (R. C. Hwa and X.-N. Wang, eds.), pp. 1-59. 2010. 0902.4041 . DOI.

[98] G. Backus and F. Gilbert, The resolving power of gross earth data, Geophysical Journal International 16 (1968) 169.

[99] A. Francis, O. Kaczmarek, M. Laine, T. Neuhaus and H. Ohno, Nonperturbative estimate of the heavy quark momentum diffusion coefficient, Phys. Rev. D92 (2015) 116003 [1508 . 04543 ].

[100] S. Caron-Huot and G. D. Moore, Heavy quark diffusion in perturbative QCD at next-to-leading order, Phys. Rev. Lett. 100 (2008) 052301 [0708.4232].

[101] H. T. Ding, A. Francis, O. Kaczmarek, F. Karsch, E. Laermann and W. Soeldner, Thermal dilepton rate and electrical conductivity: An analysis of vector current correlation functions in quenched lattice QCD, Phys. Rev. D83 (2011) 034504 [1012.4963].

[102] Y. Burnier and M. Laine, Towards flavour diffusion coefficient and electrical conductivity without ultraviolet contamination, Eur. Phys. J. C72 (2012) 1902 [1201.1994].

[103] A. Karch and E. Katz, Adding flavor to AdS / CFT, JHEP 06 (2002) 043 [hep-th/ 0205236 ].

[104] J. Erdmenger, N. Evans, I. Kirsch and E. Threlfall, Mesons in Gauge/Gravity Duals - A Review, Eur. Phys. J. A35 (2008) 81 [0711.4467].

[105] B. A. Burrington, J. T. Liu, L. A. Pando Zayas and D. Vaman, Holographic duals of flavored $N=1$ super Yang-mills: Beyond the probe approximation, JHEP 02 (2005) 022 [hep-th/ 0406207 ].

[106] C. Nunez, A. Paredes and A. V. Ramallo, Unquenched Flavor in the Gauge/Gravity Correspondence, Adv. High Energy Phys. 2010 (2010) 196714 [1002 . 1088 ].

[107] I. Kirsch and D. Vaman, The D3 / D7 background and flavor dependence of Regge trajectories, Phys. Rev. D72 (2005) 026007 [hep-th/ 0505164 ].

[108] D. Giataganas and N. Irges, Flavor Corrections in the Static Potential in Holographic QCD, Phys.Rev. D85 (2012) 046001 [1104.1623].

[109] F. Bigazzi and A. L. Cotrone, Holographic QCD with Dynamical Flavors, JHEP 01 (2015) 104 [1410.2443].

[110] T. Alho, M. Jarvinen, K. Kajantie, E. Kiritsis and K. Tuominen, On finite-temperature holographic QCD in the Veneziano limit, JHEP 01 (2013) 093 [1210 . 4516].

[111] A. F. Faedo, D. Mateos, C. Pantelidou and J. Tarrio, Towards a Holographic Quark Matter Crystal, JHEP 10 (2017) 139 [1707.06989].

[112] S.-w. Li and T. Jia, Dynamically flavored description of holographic QCD in the presence of a magnetic field, Phys. Rev. D96 (2017) 066032 [1604 .07197]. 